\title{
Impactos dos métodos de colheita da cana-de-açúcar sobre a macrofauna do solo em área de produção no Espírito Santo - Brasil
}

\section{Impacts of harvesting methods of sugar cane on the soil macrofauna in production area in Espírito Santo - Brazil ${ }^{1}$}

\author{
Eloísa dos Santos Benazzi2 ${ }^{*}$; Miriam de Oliveira Bianchi ${ }^{3}$; \\ Maria Elizabeth Fernandes Correia ${ }^{4}$; Eduardo Lima ${ }^{5}$; Everaldo Zonta ${ }^{6}$
}

\begin{abstract}
Resumo
O objetivo deste trabalho foi avaliar os efeitos dos métodos de colheita sobre a macrofauna edáfica, um conhecido indicador da qualidade do solo que detecta as alterações ocorridas no sistema e indica alternativas de manejo. O delineamento experimental adotado foi blocos casualizados com seis repetições, sendo cada bloco composto por quatro parcelas que correspondiam aos tratamentos cana crua (CC), cana queimada (CQ), cana crua-queimada (CC-Q) e cana queimada-crua (CQ-C). As coletas foram realizadas nos meses de fevereiro e julho de 2010. Os animais foram classificados em grandes grupos taxonômicos e contabilizados. Avaliaram-se os índices ecológicos (Shannon, Pielou e riqueza) e a densidade média total de indivíduos e grupos. Os dados foram analisados por ferramentas de estatística não paramétrica através do teste de Friedman ou do Sinal a 5\%. Para verificar relações entre fauna edáfica e variáveis ambientais utilizou-se um método de ordenação multivariada condicionada, a análise de redundância (RDA). O índice riqueza foi mais eficiente que a densidade média total na avaliação da influência dos sistemas de colheita da cana, estando seus maiores valores relacionados às áreas colhidas sem queima. Ainda, a ocorrência de grupos chave - também nas áreas colhidas sem queima - configura o estabelecimento de uma teia trófica. Houve dominância do grupo social Formicidae em todos os tratamentos.
\end{abstract}

Palavras-chave: Saccharum, TSBF, cana crua, cana queimada, bioindicador

\footnotetext{
Abstract

The objective of this study was to evaluate the effects of harvesting methods on the macrofauna, a known indicator of soil quality that detects changes in the system and indicates management alternatives. The experimental design was randomized blocks with six replications, with each block consisting of four parcels that corresponded to treatments green cane (CC), burnt cane (CQ), green cane - burnt cane (CC-Q) and burnt cane - green cane (CQ-C). Samples were collected in February and July 2010. The animals were divided into major taxonomic groups and accounted. Were evaluated ecological indexes (Shannon, Pielou and richness) and average total density of individuals and groups. Data were analyzed by the nonparametric statistical tools by Friedman or Signal test at $5 \%$. To check relationships between

${ }^{1}$ Parte da dissertação de mestrado da primeira autora apresentada ao Curso de Pós-Graduação em Agronomia, Ciência do Solo CPGA-CS, Universidade Federal Rural do Rio de Janeiro, UFRRJ, RJ. Apoio Capes.

${ }^{2}$ Discente de Doutorado em Ciência, Tecnologia e Inovação em Agropecuária, Universidade Federal Rural do Rio de Janeiro, UFRRJ, Seropédica, RJ. E-mail: eloisa.benazzi@gmail.com

${ }^{3}$ Discente de Doutorado em Agronomia, Ciência do Solo, UFRRJ, Seropédica, RJ. E-mail: miriambianchii@yahoo.com.br

${ }^{4}$ Pesquisadora da Embrapa Agrobiologia, Seropédica, RJ. E-mail: elizabeth.correia@embrapa.br

${ }^{5}$ Prof. Adjunto do Dept ${ }^{\circ}$ de Solos, UFRRJ, Seropédica, RJ. E-mail: ardolima2@terra.com.br

${ }^{6}$ Prof. Associado do Dept ${ }^{\circ}$ de Solos, UFRRJ, Seropédica, RJ. E-mail:ezonta@ufrrj.br

* Autor para correspondência
} 
soil fauna and environmental variables, was used a multivariate conditional ordination method, the redundancy analysis (RDA). The index richness was more efficient than the total average density to evaluate the influence of cane harvesting systems, with the highest values related to areas harvested without burning. Further, the occurrence of key groups in the areas harvested without burning configures the establishment of a trophic web. There was dominance of the social group Formicidae in all treatments.

Key words: Saccharum, TSBF, green cane, burnt cane, bioindicator

\section{Introdução}

A cana-de-açúcar é um produto vegetal de importância econômica para o Brasil, e encontra um cenário favorável no país - maior produtor mundial e detentor da tecnologia de produção de etanol de cana (BRASIL, 2012). Porém, pressões para inclusão e/ou ampliação das fontes renováveis na matriz energética mundial, com vistas à mitigação dos gases do efeito estufa, e a proibição da prática da queima no manejo da cana-de-açúcar, denotam a necessidade de avaliação dos efeitos da manutenção ou eliminação da palhada na superfície do solo.

O monocultivo impôs à cana-de-açúcar, a ocupação de pastagens, mas também a ocupação de solos férteis em regiões de topografia plana, próximos de grandes centros urbanizados, aptos para o cultivo de alimentícias. Esse sistema promove impactos ambientais que empobrecem a biodiversidade e afetam a flora e a fauna locais e regionais (ROSSETTO et al., 2010).

A fauna edáfica é afetada por fatores como: quantidade e qualidade da matéria orgânica, $\mathrm{pH}$, temperatura, umidade, textura, cobertura vegetal (SOCARRÁS, 1998), bem como as atividades agrícolas que influenciam as propriedades do solo resultando em seleção dos organismos alterando, portanto, a abundância e diversidade dos mesmos (ROSSI et al., 2009). A comunidade de minhocas, por exemplo, cai significativamente após aração (STORK; EGGLETON, 1992). Por sua íntima associação com processos ocorridos na interface serapilheira-solo, e sensibilidade aos diferentes manejos, a macrofauna reflete o quanto uma prática pode - ou não - ser conservacionista pela avaliação física e química do solo, justificando sua utilização como bioindicadora (MARTINHO et al., 2004; ROVEDDER et al., 2004; ROZANSKI et al., 2004).

Há consenso que a palha, no sistema cana-deaçúcar, constitui parte do processo de produção e interfere na manutenção de propriedades químicas, físicas, biológicas e ecológicas do solo e da cultura. As transformações dos resíduos vegetais que chegam ao solo, e sua decomposição até formas inorgânicas são resultantes da atividade de animais e microrganismos deste ecossistema. Assim, Lavelle et al. (1995) propõem um paradigma ("Paradoxo da Bela Adormecida") que explica a importância dessas interações para o ecossistema e seus mecanismos gerais, ao comparar a ação da fauna edáfica sobre a microbiota com a do Príncipe neste conto. De acordo com a proposição, comunidades microbianas - por vezes dormentes em microsítios específicos do solo - são ativadas pela passagem no tubo digestório da fauna edáfica, fator essencial para manutenção e melhora da atividade dessa comunidade.

A degradação da matéria orgânica por práticas inadequadas indica, frequentemente, depauperação da fauna de solos. De maneira inversa, práticas de manejo que garantam a manutenção da matéria orgânica favorecem uma comunidade edáfica abundante e diversificada. Dessa forma, a fauna do solo - além de apresentar variadas estratégias ecológicas - é transformadora de características físicas, químicas e biológicas, alterando a taxa e amplitude em que os processos ocorrem nos solos (LAVELLE, 2002; BARROS et al., 2004; BLANCHART et al., 2006; AQUINO et al., 2008; NUNES; ARAÚJO FILHO; MENEZES, 2009). A palhada da cana-de-açúcar apresenta maior conteúdo de materiais facilmente degradáveis, 
o que resulta em elevado teor nutricional para os organismos decompositores. $\mathrm{O}$ acúmulo da palhada corresponderia a um aporte orgânico similar ao que acontece em ambientes florestais pela deposição de serapilheira. No caso particular da colheita crua adicionam-se aspectos favoráveis como a manutenção da umidade do solo; controle de daninhas e redução no uso de herbicidas; controle da erosão; aumento da matéria orgânica à longo prazo; e redução da poluição atmosférica pela ausência de queima, à importância deste tipo de manejo no ciclo de nutrientes (PINHEIRO, 1996).

Dos organismos do solo, a macrofauna é destaque, já que seus integrantes tem o corpo em tamanho suficiente para romper estruturas de horizontes orgânicos e minerais do solo ao se alimentar, movimentar e construir galerias, aumentando assim a macroporosidade, responsável pela infiltração e percolação da água de chuva, e pela aeração dos solos (ASSAD, 1997a; GIRACCA et al., 2002). Além disso, é fundamental ao funcionamento do ecossistema, pois representa todos os níveis tróficos na cadeia alimentar do solo, afetando direta e indiretamente a produção primária (SILVA et al., 2007). Lavelle et al. (1994) discutindo o tema fauna do solo inferiu que esta pode ser manipulada para melhorar propriedades físicas do solo e regular processos de decomposição da matéria orgânica. Portanto, os invertebrados não são habitantes passivos do solo, mas sim constituintes ativos e integrais (STORK; EGGLETON, 1992).

Neste sentido, a queima do canavial afeta a biota do solo associada à cultura, diretamente, pelo fogo ou, indiretamente, pela remoção da palhada que protege o solo expondo-o a variações de temperatura e umidade, por meio da incidência solar, devido as maiores taxas de evaporação e evapotranspiração. A queima, eliminando a cobertura do solo, altera condições ambientais, de abrigo, e de alimentação limitando os nichos ecológicos (ARAÚJO FILHO; BARBOSA, 2000), ou seja, as condições de sobrevivência da fauna edáfica. Em oposição à queima, a colheita da cana- de-açúcar crua contribui para a manutenção de adequada umidade, temperatura e matéria orgânica do solo, promovendo a melhoria do ambiente e induzindo maior ocorrência de organismos pela oferta de recursos alimentares e/ou habitats. O que se dá pela deposição da palhada que aumenta a probabilidade de saprófagos encontrarem alimento durante todo o ano, além de gerar uma estrutura de micro habitats que possibilitam a colonização dos organismos edáficos (MAJER, 1984; ALMEIDA FILHO, 1995; COLLETT, 1998; TOPP et al., 2001; CAMPANHOLA, 2002; WANNER; DUNGER, 2002). Pontualmente, a cultura implantada e a manutenção da palhada afetam as comunidades edáficas (BATÁRY et al., 2008), destacando-se a macrofauna saprófaga, os microrganismos e as relações predador-presa.

Pela impossibilidade de se avaliar uma comunidade edáfica integralmente, retratamse parcelas escolhendo determinados grupos taxonômicos associados a frações do habitat, ou grupos que tenham função semelhante no ecossistema. Qualquer que seja a abordagem de estudo é preciso duas ferramentas básicas: a abundância - que fornece uma visão parcial da comunidade - e a diversidade de espécies ou grupos presentes (DANGERFIELD; TELFORD, 1992; MERLIM, 2005). O conhecimento dos grupos da fauna do solo e das funções por eles desempenhadas (fragmentação, controle da população microbiana, alterações estruturais de habitat) permite um manejo direto (inserção de grupos em um ambiente) ou indireto (favorecimento de habitats) (SNYDER; HENDRIX, 2008).

Nesse sentido, o estudo da fauna assume grande importância no processo produtivo das plantas cultivadas, devido ao papel fundamental que ocupa no subsistema decompositor. Desta forma, o objetivo deste estudo é avaliar os impactos do métodos de colheita da cana-de-açúcar sobre a macrofauna edáfica em áreas de produção, em diferentes períodos climáticos. 


\section{Material e Métodos}

O estudo foi conduzido em um Argissolo Amarelo, na usina Linhares Agroindustrial S.A. (LASA), localizada no município de LinharesES, nas coordenadas geográficas 19²3'48'S e $40^{\circ} 03^{\prime} 42^{\prime}$ W. A vegetação primária remanescente é representada por uma floresta tropical subperenifólia e altitude local de $28 \mathrm{~m}$. O clima regional é o tropical úmido com período chuvoso no verão e seco no inverno, Aw pela classificação de Köppen. Nos anos de 2009 e 2010 o regime hídrico foi atípico (Figura 1).

Figura 1. Temperaturas médias mínimas e máximas e precipitação no município de Linhares - ES mensais para os anos de 2009 e 2010. Fonte: INCAPER (2012).

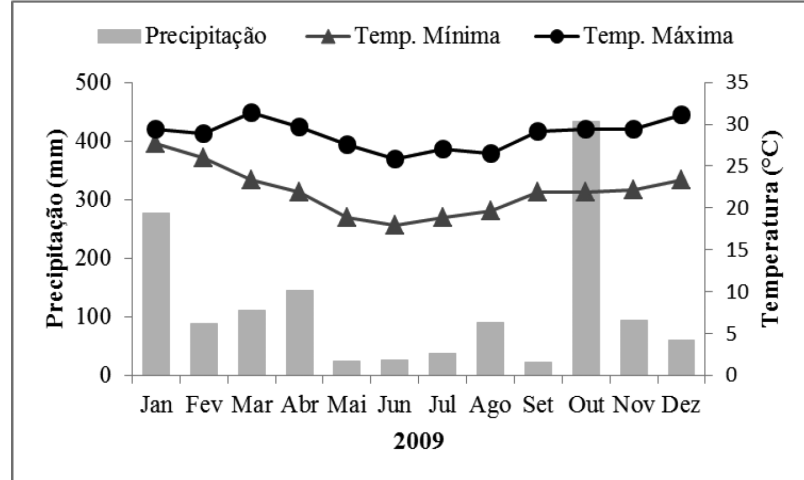

Fonte: Elaboração dos autores.

O experimento, de longa duração, foi instalado em maio de 1989 em área de tabuleiros em solo classificado como Argissolo Amarelo distrófico textura arenosa/média (RAVELLI NETO; LIMA, 1987; EMBRAPA, 2006), sendo a última renovação da área em 2005. O delineamento experimental utilizado é o de blocos ao acaso, sendo seis blocos contendo quatro parcelas. Cada uma delas possui $286 \mathrm{~m}^{2}$, com 11 linhas de $20 \mathrm{~m}$ de comprimento, espaçadas de $1,30 \mathrm{~m}$. A implantação da cultura (cultivar SP79-1011) foi feita utilizando-se o cultivo mínimo e a adubação utilizada foi uniforme, seguindo a rotina da usina, sendo aplicados no plantio, em 17/05/2005, $500 \mathrm{~kg} \mathrm{ha}^{-1}$ da formulação 05-20-20, e na soca, que ocorre - anualmente nos meses de outubro/novembro, $500 \mathrm{~kg} \mathrm{ha}^{-1}$ da formulação 20-00-30.

Os tratamentos são as formas de renovação do canavial, onde, em uma das parcelas a colheita

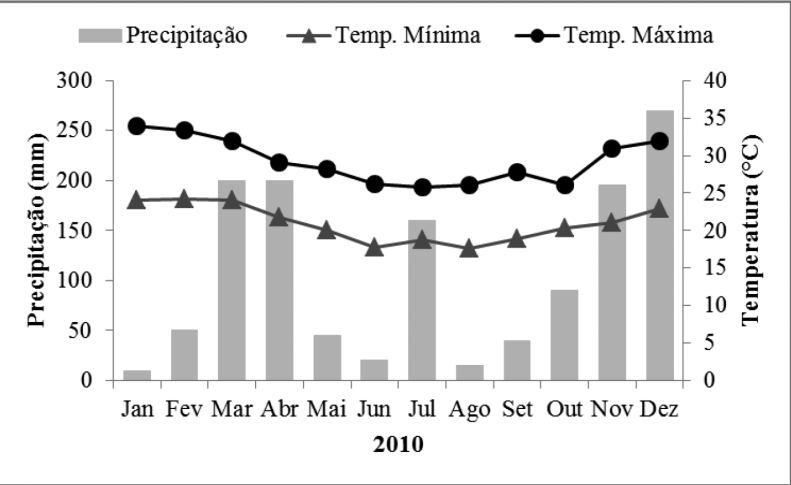

manual ocorre sem queima (CC), e em outra, com queima (CQ). Nas duas restantes, se alterna por ocasião da renovação cana crua/cana queimada (CC-Q) e cana queimada/cana crua (CQ-C). As colheitas ocorrem, geralmente, no mês de setembro. As amostragens de fauna edáfica foram realizadas nas entrelinhas da cultura, nos meses de fevereiro e julho de 2010, mas, considerando o regime hídrico atípico durante este ano, preferiu-se não inferir o efeito da sazonalidade (período seco ou chuvoso), havendo apenas a discriminação dos meses como as épocas de coleta.

A macrofauna foi coletada utilizando-se o método dos monólitos de solo recomendado pelo Programa Tropical Soil Biology and Fertility - TSBF, com modificações (LAVELLE, 1988; ANDERSON; INGRAM, 1993). Em cada parcela foi amostrado um monólito de solo de 25 x 25 $\mathrm{cm}$ tendo-se, ao final, seis repetições. Coletou- 
se primeiro a amostra da serrapilheira contida na área demarcada pelo gabarito, e em seguida o solo localizado na projeção do gabarito metálico até 30 $\mathrm{cm}$ de profundidade. As amostras de solo foram subdivididas em camadas de $10 \mathrm{~cm}$, destorroadas e reviradas manualmente em bandejas. Com uma pinça foi feita a captura dos organismos que foram acondicionados em recipientes etiquetados contendo solução preservativa: álcool 70\% e formol 4\% para Oligochaetas. A contagem e identificação da fauna do solo ocorreram em laboratório e a identificação, com auxílio de lupa binocular, se deu em nível de grandes grupos taxonômicos (DEVIDE; CASTRO, 2008).

Para a avaliação da diversidade e abundância das comunidades edáficas utilizaram-se os seguintes estimadores: o índice de Shannon $(\mathrm{H}=-\Sigma$ pi log pi, onde $\mathrm{pi}=\mathrm{ni} / \mathrm{N} ; \mathrm{ni}=$ densidade de cada grupo; $\mathrm{N}$ = somatório do número de indivíduos por metro quadrado); a riqueza total (número de grupos nos diferentes tratamentos); a riqueza média (número médio de grupos presentes em cada tratamento); o índice de Pielou (e $=\mathrm{H} \log \mathrm{S}^{-1}$, onde $\mathrm{H}=$ índice de Shannon; $\mathrm{S}=$ riqueza total); e as densidades dos diferentes grupos e do total de indivíduos por tratamento e amostragem (ind $\mathrm{m}^{-2}$ ), associadas aos respectivos erros padrões. Os dados foram submetidos à estatística não paramétrica, sendo usado para a comparação entre tratamentos o teste de Friedman a $5 \%$ de probabilidade e, para a comparação entre coletas, o do Sinal a 5\% de probabilidade, com as ferramentas contidas no programa Statistica 7.0. Para verificar as relações entre fauna edáfica e variáveis ambientais foi utilizado um método de ordenação multivariada condicionada, a análise de redundância (RDA), mais apropriado quando as variáveis exibem relações lineares com os gradientes ambientais (LEGENDRE; ANDERSON, 1999). Para testar a significância das relações foi utilizado o teste de Monte-Carlo. As análises foram realizadas com o programa CANOCO 4.5, e o diagrama de ordenação confeccionado com as ferramentas do programa Cano Draw (ter BRAAK; SMILAUER, 2002).

\section{Resultados e Discussão}

As densidades médias totais variaram de 20 ind. $\mathrm{m}^{-2}$ para o tratamento com queima (CQ), em fevereiro, à 839 ind. $\mathrm{m}^{-2}$ no tratamento cana crua (CC), no mês de julho, coincidindo com os manejos mais antigos (Tabela 1). Considerando apenas as sucessões cana crua-queimada e cana queimadacrua (CC-Q e CQ-C), as densidades variaram de 85 ind $\mathrm{m}^{-2}$ (fevereiro) a 628 ind $\mathrm{m}^{-2}$ (julho), ambas para o tratamento CC-Q. Foram observadas diferenças significativas entre tratamentos, em fevereiro/2010, para a densidade média total da macrofauna, onde os tratamentos CQ-C e CQ apresentaram, respectivamente, a maior e a menor densidade. Em relação à amostragem de julho/2010, não foram verificadas diferenças significativas na abundância entre tratamentos. Na comparação entre épocas de coleta, apenas o tratamento CQ diferiu estatisticamente, sendo os maiores valores encontrados para a coleta de julho/2010. Ao avaliar cultivos de cana-de-açúcar colhidos com e sem queima, Pinheiro (1996) encontrou as maiores densidades de organismos edáficos em áreas com colheita crua, concluindo que esta colheita além de favorecer a fauna em quantidade, o faz também em riqueza de grupos.

Dlamini e Haynes (2004) avaliando os efeitos de diferentes manejos e cultivos sobre a população de Oligochaetas em Kwazulu-Natal (África) encontraram maior número de indivíduos em áreas de mata, seguindo-se da cana crua e por fim a área de cana queimada, o que está de acordo com o obtido por Ceddia (1996) que também encontrou maior número de indivíduos na área sem queima. Avaliando a influência do método de colheita e da aplicação de vinhaça sobre a macrofauna edáfica no Paraná, Pasqualin et al. (2012) descreram valores de densidade (médios de seis coletas) da ordem de 182 ind $\mathrm{m}^{-2}$ em cana crua sem vinhaça e 137 ind $\mathrm{m}^{-2}$ em cana queimada sem vinhaça. Dessa forma, para este estudo, a abundância total de indivíduos apresenta valores abaixo e acima, respectivamente, dos anteriormente citados. 
Tabela 1. Densidade média total da macrofauna edáfica ${ }^{(1)}\left(\right.$ ind $\left.^{-2}\right)$ nos diferentes tratamentos, para ambas as épocas de coleta.

\begin{tabular}{ccccc}
\hline Coletas & \multicolumn{4}{c}{ Ind m ${ }^{-2} \pm$ Erro Padrão } \\
\hline & $\mathrm{CC}$ & $\mathrm{CQ}$ & CC-Q & CQ-C \\
Fevereiro 2010 & $173 \pm 69 \mathrm{Aab}$ & $20 \pm 5 \mathrm{Bb}$ & $85 \pm 50 \mathrm{Aab}$ & $287 \pm 112 \mathrm{Aa}$ \\
Julho 2010 & $839 \pm 422 \mathrm{Aa}$ & $328 \pm 248 \mathrm{Aa}$ & $628 \pm 490 \mathrm{Aa}$ & $437 \pm 175 \mathrm{Aa}$ \\
\hline
\end{tabular}

$\mathrm{CC}$ - cana crua $\quad \mathrm{CQ}$ - cana queimada $\quad \mathrm{CC}-\mathrm{Q}$ - cana crua-queimada $\quad \mathrm{CQ}-\mathrm{C}$ - cana queimada-crua

(1) Médias de seis repetições. Valores seguidos de mesma letra maiúscula, na linha, não diferem entre si pelo teste de Friedman a 5\% de probabilidade. Valores seguidos de mesma letra minúscula, na coluna, não diferem entre si pelo teste do Sinal a 5\% de probabilidade.

Fonte: Elaboração dos autores.

Analisando os índices ecológicos (Tabela 2) para fevereiro, os tratamentos CC e CQ-C apresentaram valores significativamente maiores de riqueza média. A maior diversidade, verificada pelo índice de Shannon, foi encontrada para o tratamento CQ$\mathrm{C}$, assim como os maiores valores de densidade, riqueza média e total, uma vez que este índice reflete a riqueza dos grupos. Isso indica que, na amostragem, o tratamento oferecia ambiente favorável à atividade da fauna quanto aos recursos: alimentar, microclima e abrigo. Ou seja, a manutenção da cobertura do solo condicionou a ocorrência de diferentes grupos da fauna. Considerando que, desde 2005, não há renovação da área, a proteção contra o impacto direto das gotas de chuva e erosão, assim como a manutenção da umidade - garantidas pela cobertura do solo - constituem os pontos de maior importância e influência aos distintos grupos edáficos. Os tratamentos com queima apresentaram índices de diversidade baixos, semelhante àqueles observados por Pasqualin et al. (2012). De acordo com Souza et al. (2005), a menor diversidade de organismos edáficos está associada à exposição do solo a variações de temperatura, perda de umidade e a menor oferta de alimento e abrigo para alguns grupos, o que afeta as comunidades mais sensíveis e, consequentemente, a diversidade.

Em julho/2010, no tratamento CQ-C, o índice de Pielou foi baixo, apesar do maior valor para o índice de Shannon. Mesmo sendo a densidade e riqueza elevadas, há dominância de grupos, resultando em maior valor apenas de Shannon. O índice de Pielou apresentou seu maior valor para o tratamento CC-Q (Tabela 2), logo não há dominância entre grupos, uma vez que este tratamento apresenta densidades muito baixas, caracterizando uma melhor distribuição dos organismos nos poucos grupos encontrados. Tal resultado é corroborado pelo exposto por Correia e Oliveira (2000) de que a queima tem efeitos negativos sobre a fauna do solo, pois além da eliminação direta dos animais que vivem na superfície, a remoção da serrapilheira elimina a fonte alimentar e desestrutura o habitat. Sem alimento e sem habitat, a recolonização, quando ocorre, é lenta e restrita a poucos grupos. Menezes et al. (2009) explicam que nem sempre a comunidade com maiores valores para o índice de Pielou será a mais diversa por, indiretamente, haver menor dominância de poucas espécies ou grupos. Para isso, citam a afirmação de Gosselin (2006), de que embora a equitabilidade seja um componente da diversidade estruturado para ser independente da riqueza, ele não o é. Essa relação é forte, principalmente quando a riqueza é inferior a 20, variando muitas vezes de maneira inversa à riqueza. Para a cana queimada (CQ) isto pode ser observado, uma vez que o tratamento foi o de menor riqueza média, total e densidade, e maior equitabilidade. 
Tabela 2. Índices ecológicos em função do tratamento.

\begin{tabular}{lcccc}
\hline Tratamento & Riqueza Média $^{(1)}$ & Riqueza Total & Índice de Shannon & Índice de Pielou \\
\hline Fevereiro de 2010 & & & & \\
\hline CC & $3 \mathrm{Aa}$ & 7 & 1,53 & 0,54 \\
CQ & $1 \mathrm{Ba}$ & 3 & 1,38 & 0,87 \\
CC-Q & $1 \mathrm{Aab}$ & 3 & 0,79 & 0,50 \\
CQ-C & $3 \mathrm{Aa}$ & 9 & 1,79 & 0,57 \\
\hline Julho de 2010 & & & & \\
\hline CC & $3 \mathrm{Aa}$ & 9 & 0,49 & 0,15 \\
CQ & $3 \mathrm{Aa}$ & 8 & 0,76 & 0,25 \\
CC-Q & $2 \mathrm{Aa}$ & 6 & 0,99 & 0,38 \\
CQ-C & $4 \mathrm{Aa}$ & 11 & 1,14 & 0,33 \\
\hline
\end{tabular}

CC - cana crua $\quad$ CQ - cana queimada $\quad$ CC-Q - cana crua-queimada CQ-C - cana queimada-crua

(1) Médias de seis repetições. Valores seguidos de mesma letra maiúscula, na linha, não diferem entre si pelo teste de Friedman a 5\% de probabilidade. Valores seguidos de mesma letra minúscula, na coluna, não diferem entre si pelo teste do Sinal a 5\% de probabilidade.

Fonte: Elaboração dos autores.

Para o mês de julho observam-se maiores abundâncias de indivíduos em todos os tratamentos avaliados (Tabela 1). Isso pode estar relacionado ao fato de, até fevereiro, o experimento ter passado por estresses hídricos extremos enfrentando chuvas intensas seguidas de seca prolongada, afinal o tamanho das populações amostradas é resultado de eventos climáticos anteriores à coleta (CORREIA; PINHEIRO, 1999), de uma maior cobertura vegetal do solo, bem como da maior umidade verificada em campo. Contudo, as maiores densidades se devem a presença do grupo social Formicidae que chega a representar $94 \%$ do total de indivíduos em CC. Correia e Pinheiro (1999) ao monitorarem a fauna do solo em diferentes coberturas vegetais em sistema integrado de produção agroecológica observaram que, independentemente da cobertura, as menores densidades da fauna ocorreram em ano, ou períodos, de déficit hídrico. O mesmo padrão foi observado por Moço et al. (2005) que ao caracterizar a fauna em diferentes coberturas vegetais obtiveram maiores densidades de organismos no verão. Na segunda coleta (Julho/2010), não houve diferenças significativas entre tratamentos para a riqueza média. A riqueza total seguiu o padrão observado anteriormente. Já os índices de diversidade e equitabilidade foram baixíssimos, podendo ser atribuídos à dominância de Formicidae, o mesmo foi observado por Pasqualin et al. (2012) em áreas de cana-de-açúcar sob diferentes manejos de colheita e aplicação de vinhaça.

Elevadas densidades do grupo Formicidae não implicam em uma estrutura complexa de comunidade, pois são organismos que se adaptam às condições locais, predominando uma ou poucas espécies (SILVA et al., 2006), sendo capazes de colonizar áreas em detrimento de outros grupos edáficos que não colonizam, ou migram para locais com melhores condições de sobrevivência. Alves et al. (2008) observaram que a alta frequência de Formicidae pode ser resultado de áreas mal manejadas, enquanto Nunes, Araújo Filho e Menezes (2008) afirmam que a ocorrência em maiores proporções indicaria o equilíbrio das áreas de ocorrência, pois os Formicidae ocupam nichos diversificados no ecossistema (SILVA; BRANDÃO, 1999). A presença em distintas condições se deve ao fato de atuarem no transporte de nutrientes e na dinâmica da matéria orgânica; de indicarem alterações no manejo do solo, principalmente durante a reabilitação; ou devido à disponibilidade de alimento se relacionar à abundância de formigas (BARETTA et al., 2006; PARR et al., 2007; 
SANTOS et al., 2012). Sendo comum, em ambientes impactados (ex. áreas de mineração e pastagens degradadas), que algumas espécies de formigas assumam papel de colonizadoras (MAJER, 1996; ANDERSEN et al., 2002; DIAS et al., 2006). Ainda, as altas densidades do grupo, em todas as áreas, seriam explicadas pelo método de amostragem, que privilegia a captura de insetos sociais (MERLIM, 2005).

O total de indivíduos coletados em fevereiro foi identificado em dez grupos taxonômicos (Tabela 3). Desses, sete foram verificados na CC, três em CQ e CC-Q e nove na CQ-C. Apenas Formicidae e larvas de Coleoptera ocorreram nos quatro tratamentos, sendo justamente os de maior densidade. Não foram verificadas diferenças significativas entre tratamentos. Em julho, foram identificados treze grupos taxonômicos (Tabela 4) sendo nove encontrados em CC, oito em CQ, seis em CC-Q e onze em CQ-C. Somente Formicidae e Oligochaeta apareceram em todos os tratamentos. Araneae apresentou diferença significativa entre tratamentos, sendo sua densidade maior no tratamento CQ$\mathrm{C}$, quando comparado aos demais, o que pode ser atribuído ao fato deste ser um grupo com maior resiliência as modificações ambientais (PINHEIRO, 1996). A presença de predadores (Araneae e Chilopoda) nos tratamentos CC e CQ-C indica um ambiente mais estável e o estabelecimento de uma cadeia alimentar, pois seriam estes os tratamentos que ofereceriam uma gama de presas. Já a maior participação do grupo Oligochaeta pode estar relacionado à maior umidade observada em campo, a alterações qualitativas na palhada e ao aporte de matéria orgânica também em subsuperfície via sistema radicular.

Tabela 3. Distribuição da densidade da macrofauna ${ }^{(1)}\left(\right.$ ind $\left.\mathrm{m}^{-2}\right)$ por grupos para o mês de fevereiro/2010.

\begin{tabular}{|c|c|c|c|c|c|c|c|c|}
\hline \multirow{2}{*}{ Grupos Taxonômicos } & \multicolumn{2}{|c|}{$\mathrm{CC}$} & \multicolumn{2}{|c|}{ CQ } & \multicolumn{2}{|c|}{ CC-Q } & \multicolumn{2}{|c|}{ CQ-C } \\
\hline & \multicolumn{8}{|c|}{----------------------- Ind m ${ }^{-2}$---------------------- } \\
\hline Araneae & 11 & A & 0 & A & 5 & A & 3 & A \\
\hline Auchenorryncha & 3 & A & 3 & A & 0 & A & 0 & A \\
\hline Blattodea & 3 & A & 0 & A & 0 & A & 5 & A \\
\hline Coleoptera & 8 & A & 0 & A & 0 & A & 5 & A \\
\hline Formicidae & 117 & A & 11 & A & 69 & A & 171 & A \\
\hline Gastropoda & 0 & A & 0 & $\mathrm{~A}$ & 0 & $\mathrm{~A}$ & 19 & A \\
\hline Isoptera & 0 & A & 0 & $\mathrm{~A}$ & 0 & $\mathrm{~A}$ & 64 & A \\
\hline Coleoptera (larvas) & 27 & A & 5 & $\mathrm{~A}$ & 8 & A & 13 & $\mathrm{~A}$ \\
\hline Oligochaeta & 3 & A & 0 & A & 0 & $\mathrm{~A}$ & 3 & A \\
\hline Sternorryncha & 0 & A & 0 & A & 0 & $\mathrm{~A}$ & 3 & A \\
\hline
\end{tabular}

$\mathrm{CC}$ - cana crua $\quad \mathrm{CQ}$ - cana queimada $\quad \mathrm{CC}-\mathrm{Q}$ - cana crua-queimada $\quad \mathrm{CQ}-\mathrm{C}$ - cana queimada-crua

(1) Médias de seis repetições. Valores seguidos de mesma letra maiúscula, na coluna, não diferem entre si pelo teste de Friedman a $5 \%$ de probabilidade.

Fonte: Elaboração dos autores. 
Tabela 4. Distribuição da densidade da macrofauna ${ }^{(1)}\left(\right.$ ind $^{-2}$ ) por grupos para o mês de julho/2010.

\begin{tabular}{|c|c|c|c|c|c|c|c|c|}
\hline \multirow{2}{*}{ Grupos Taxonômicos } & \multicolumn{2}{|c|}{$\mathrm{CC}$} & \multicolumn{2}{|c|}{ CQ } & \multicolumn{2}{|c|}{ CC-Q } & \multicolumn{2}{|r|}{ CQ-C } \\
\hline & \multicolumn{8}{|c|}{ 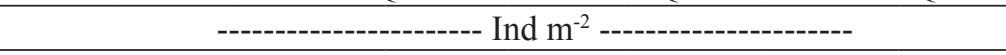 } \\
\hline Araneae & 16 & $\mathrm{AB}$ & 0 & B & 0 & B & 19 & A \\
\hline Auchenorryncha & 0 & A & 0 & A & 0 & A & 3 & A \\
\hline Blattodea & 5 & A & 3 & A & 0 & A & 8 & A \\
\hline Oligochaeta (casulo) & 11 & A & 3 & A & 3 & A & 0 & A \\
\hline Chilopoda & 3 & A & 0 & A & 0 & A & 3 & A \\
\hline Coleoptera & 3 & A & 5 & A & 0 & A & 5 & A \\
\hline Formicidae & 787 & A & 293 & A & 464 & A & 363 & A \\
\hline Gastropoda & 0 & A & 3 & A & 0 & A & 3 & A \\
\hline Heteroptera & 0 & A & 0 & A & 3 & A & 3 & A \\
\hline Isoptera & 0 & A & 8 & A & 147 & A & 0 & A \\
\hline Coleoptera (larvas) & 3 & A & 0 & A & 0 & A & 5 & A \\
\hline Oligochaeta & 8 & $\mathrm{~A}$ & 11 & A & 8 & A & 11 & A \\
\hline
\end{tabular}

CC - cana crua $\quad \mathrm{CQ}$ - cana queimada $\quad \mathrm{CC}-\mathrm{Q}$ - cana crua-queimada $\mathrm{CQ}-\mathrm{C}$ - cana queimada-crua

(1) Médias de seis repetições. Valores seguidos de mesma letra maiúscula, na coluna, não diferem entre si pelo teste de Friedman a $5 \%$ de probabilidade.

Fonte: Elaboração dos autores.

A não diferenciação entre tratamentos do grupo Formicidae seria influenciada pelo fato destes indivíduos serem extremamente móveis, transitando de um local a outro. Esses resultados estão de acordo com os apresentados por Cordeiro et al. (2004) que avaliando macrofauna em um sistema de manejo orgânico de produção, não verificaram diferenças significativas entre tratamentos, mesmo sendo os Formicidae o grupo de maiores densidade e dominância, atribuindo isso tanto a proximidade entre as áreas, quanto à mobilidade do grupo. Pinheiro (1996), também avaliando a macrofauna em área de cana-de-açúcar colhida com e sem queima, encontrou maiores densidades para o grupo Formicidae em ambas as áreas. Ainda, Pasqualin et al. (2012) verificaram o predomínio de Formicidae em áreas de cana-de-açúcar. A ampla ocorrência deste grupo pode ser atribuída ao hábito social, a repartição do trabalho e ao hábito alimentar diversificado em função da espécie, sendo importantes em processos de decomposição dos ecossistemas tropicais (ASSAD, 1997b; BRUYN, 1999). Barrios et al. (2005) consideram que densidades elevadas, especificamente de Formicidae, correspondem à uma situação em que a dominância desse grupo se daria em solos onde, anteriormente, houve algum processo de degradação, mesmo que o manejo atual seja de recuperação. Para Kitamura et al. (2008), o surgimento das formigas como pioneiras sugere que esse grupo é indicador de estresse, por colonizar as áreas quando estas não apresentam condições adequadas de sobrevivência, o que também justificaria seus maiores valores para os tratamentos submetidos a queima.

Não houve diferenças significativas para os grupos observados, em função do tratamento, entre coletas pelo teste do Sinal a 5\% de probabilidade. Entre as camadas de solo avaliadas houve diferença significativa apenas para o grupo Formicidae, na profundidade de $0-10 \mathrm{~cm}$ do tratamento CC. Durante todo o estudo este grupo se destacou, apresentando as maiores densidades e sendo responsável pela menor equitabilidade dos grupos, mesmo diante de uma comunidade mais diversa. Bianchi, Aquino e Almeida (2007) e Pasqualin et al. (2012) reforçam que, em áreas cultivadas, a fauna tem preferência pelas camadas superficiais de solo. Altas densidades refletem a preferência do grupo pela camada de solo que se diferencia das demais. 
A distribuição vertical da fauna nos a habitar as camadas mais profundas do solo compartimentos do solo (Figura 2), no período fevereiro/2010, mostra que a densidade de organismos encontrada foi dependente do tratamento, sendo que para os tratamentos $\mathrm{CC}$ e CQ-C cerca de 50 e $60 \%$, respectivamente, do número total de indivíduos concentraram-se na camada de $0-10 \mathrm{~cm}$. No tratamento CQ houve maior concentração de indivíduos na camada de $10-20 \mathrm{~cm}$, enquanto no tratamento CC-Q o maior percentual de indivíduos estava na camada inferior $(20-30 \mathrm{~cm})$. O gradiente de distribuição vertical, isto é, a menor concentração de indivíduos nas camadas superiores ocorre por influencia direta da queima e é agravado pelo fato da área experimental ter enfrentado regime hídrico atípico, com período de seca, antes da realização da coleta (Figura 1). Ademais, a baixa umidade afeta muitos animais, devido as suas características morfológicas (VERHOEF; WITTEVEEN, 1980; DIDDEN, 1993). Assim, buscando condições amenas, os animais passam (DIDDEN, 1993), ou até mesmo migram para áreas com melhores condições de sobrevivência. As constantes variações e, principalmente, a falta de umidade no solo afetam a fauna por influenciar a comunidade microbiana, visto que alguns grupos utilizam microrganismos como fonte alimentar (HÅGVAR, 1998). Dessa forma, percebe-se que nos primeiros $10 \mathrm{~cm}$ de profundidade, para tratamentos em que ocorre a manutenção da cobertura do solo, é onde se concentra a maior densidade de organismos edáficos. Tão importante quanto o efeito da seca é o fato de em áreas onde se utiliza o fogo como método pré-colheita haver, durante a queima, elevação da temperatura nos primeiros centímetros de solo eliminando os organismos, a fonte alimentar e promovendo alterações no habitat, gerando assim gradiente de distribuição dos animais, uma vez que maiores densidades são esperadas nas camadas superficiais do solo.

Figura 2. Distribuição vertical da macrofauna edáfica para a coleta de fevereiro/2010.

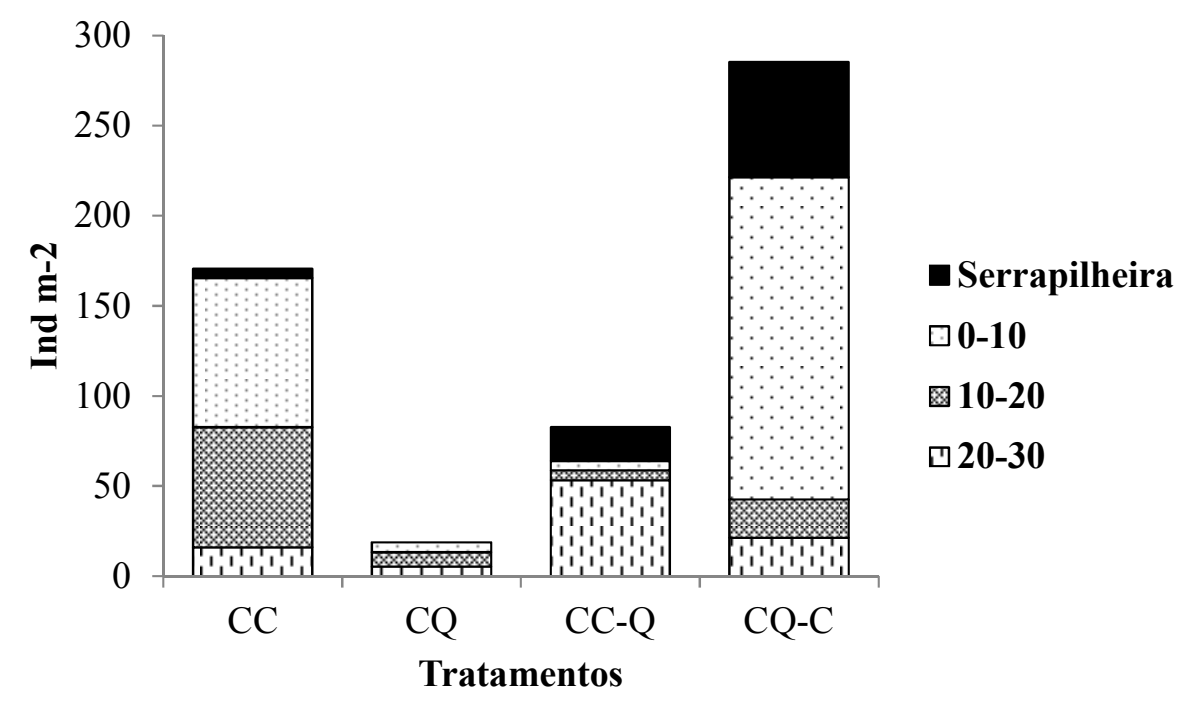

CC - Cana crua $\quad$ CQ - Cana queimada CC-Q - Cana crua-queimada CQ-C - Cana queimada-crua.

Fonte: Elaboração dos autores. 
Para a coleta de julho foram detectadas diferenças significativas entre as camadas avaliadas apenas para o grupo Formicidae, sendo os maiores valores encontrados para a profundidade de $0-10 \mathrm{~cm}$ no tratamento CC-Q, que na época amostral apresentou a segunda maior densidade média total. Dessa forma, confirma-se que em ambas as coletas, o grupo de maior influência na dinâmica da fauna do solo, bem como sujeito aos efeitos do clima e manejo foi o Formicidae. A distribuição vertical da fauna nos compartimentos do solo (Figura 3), em julho/2010, mostra que a densidade de organismos encontrada foi dependente de outros fatores, possivelmente, ambientais os quais não foram contemplados no estudo, não havendo influência dos tratamentos. Tal distribuição foi, ainda, função dos grupos mais expressivos, uma vez que para o tratamento $\mathrm{CC}$, o maior percentual de indivíduos - cerca de $60 \%$ - se encontra na camada de 20-30 cm, correspondendo aos indivíduos do grupo Formicidae.

Figura 3. Distribuição vertical da macrofauna edáfica para a coleta de julho/2010.

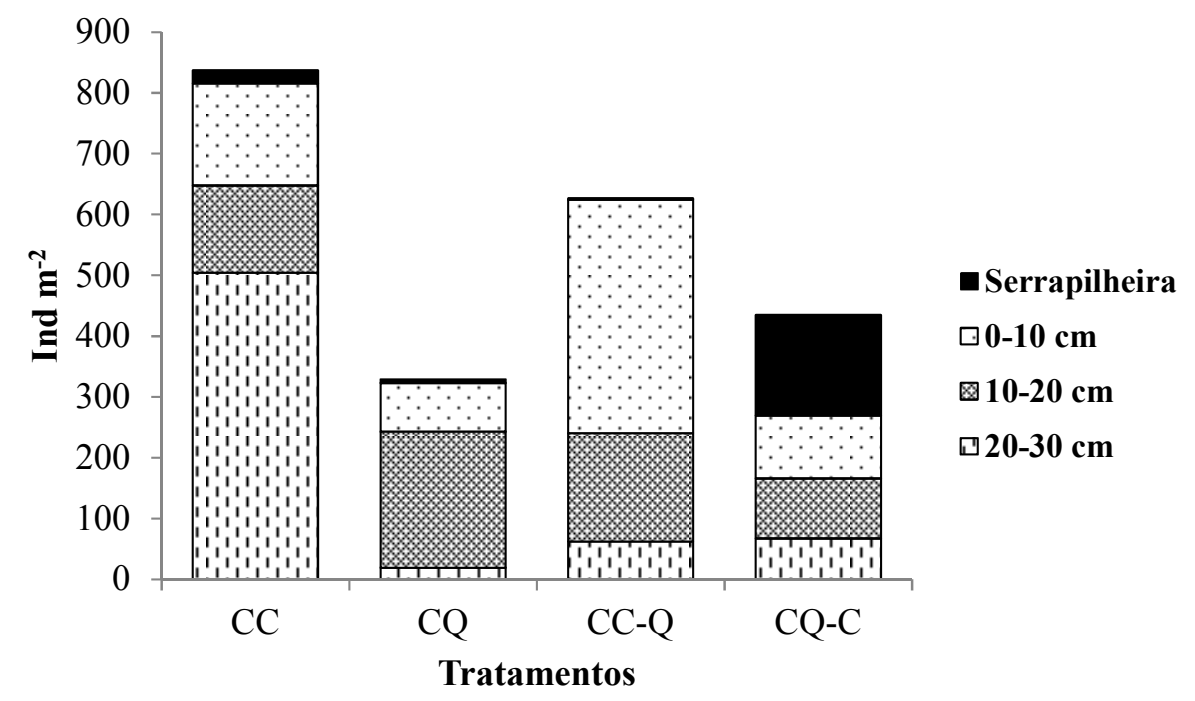

CC - Cana crua

CQ - Cana queimada

CC-Q - Cana crua-queimada

CQ-C - Cana queimada-crua.

Fonte: Elaboração dos autores.

No tratamento CQ houve maior concentração de indivíduos na camada de 10-20 cm, enquanto no tratamento CC-Q o maior percentual de indivíduos estava na camada de $0-10 \mathrm{~cm}$, novamente um reflexo da dominância de Formicidae e, dessa vez, também de Isoptera cujas densidades corresponderam a, aproximadamente, $60 \%$ do total de indivíduos encontrados nesse tratamento, para a camada de solo. Para o tratamento CQ-C fica evidente o benefício da manutenção da palhada no solo, pois cerca de $40 \%$ dos indivíduos totais foram observados na interface solo-serrapilheira. As diferenças observadas para a densidade média total e a distribuição vertical entre as datas de estudo refletem, provavelmente, alterações qualitativas na serrapilheira determinando diferenças na colonização ocorrida neste material pelas populações edáficas, conforme observado por Pinheiro (1996). As avaliações utilizando como artifício a relativização das densidades de cada grupo presente nas amostragens, isto é, suas contribuições percentuais na densidade média total dos tratamentos permitem a visualização daqueles grupos de maior expressividade. De modo geral, este posto foi ocupado pelo grupo Formicidae, que 
exerceu dominância sobre os demais grupos em todos os tratamentos para ambas as épocas de coleta.

Para o tratamento CQ-C há uma melhor distribuição dos indivíduos entre as camadas do solo, o que decorre do maior conteúdo de matéria orgânica, inclusive em profundidade devido ao sistema radicular, de microclimas e oferta de refúgios que favorecem a atividade da fauna e estimulam o surgimento de outros grupos com diferentes estratégias de sobrevivência. Pinheiro (1996) afirma que a macrofauna tem preferência pela serrapilheira e que o solo é influenciado tanto pelo material em superfície quanto pela fauna que a transforma, já que a eliminação desta parece afetar a fauna da primeira camada de solo, não desconsiderando o efeito do fogo. Ainda, certos padrões quanto aos grupos funcionais da macrofauna edáfica foram observados em relação aos tratamentos. Em fevereiro, os tratamentos CC, CQ e CQ-C contaram com a presença de fitófagos (Auchenorryncha e Sternorryncha). Já organismos tidos como sendo predadores foram observados nos tratamentos CC, CC-Q e CQ-C (Araneae), e aqueles grupos enquadrados como saprófagos (Blattodea, Gastropoda e Oligochaeta), estavam relacionados às áreas com manutenção de cobertura.

Em julho, época em que se observou maiores densidades médias totais de indivíduos, os predadores estiveram presentes, exclusivamente, nos tratamentos CC e CQ-C (Araneae e Chilopoda). A presença de grupos predadores nos tratamentos cuja palha é mantida em superfície implica em duas caracterizações: i) a do estabelecimento de uma teia trófica, uma vez que para que indivíduos deste grupo existam é necessário haver a colonização da área pelos predecessores na cadeia alimentar e, consequentemente, a de um ambiente mais estável (equilibrado); ii) outro indicativo de um ambiente melhor estruturado é a diversidade dos grupos predadores.

Os organismos considerados fitófagos (Auchenorryncha e Heteroptera) foram constatados nos tratamentos CC-Q e CQ-C. Já os demais grupos funcionais observados foram os saprófagos (Blattodea, Gastropoda e Oligochaeta), que nessa amostragem encontraram-se associados, não só aos tratamentos sem queima da palhada, mas também aos demais tratamentos. Tal associação pode estar relacionada à deposição, de folhas senescentes, inclusive nas áreas queimadas, e a presença de diversos materiais orgânicos dos quais esses organismos edáficos se alimentam que foram disponibilizados pela ação da macrofauna que anteriormente colonizou aquelas áreas melhorando, assim, condições ambientais e possibilitando o estabelecimento de outros indivíduos. Os grupos Oligochaeta e Formicidae foram encontrados em todos os tratamentos.

As variáveis ambientais testadas, que correspondem às épocas (fevereiro e julho) e aos manejos de colheita da cana-de-açúcar explicam $16,4 \%$ de toda a variação da comunidade da macrofauna do solo (Figura 4). Indicando que os padrões de ocorrência, abundância e diversidade dos invertebrados do solo na área sofrem grande influência de outras variáveis ambientais não consideradas neste estudo. No entanto, no que concerne às variáveis ambientais avaliadas, a variância explicada pela relação com a fauna, para a soma dos dois primeiros eixos, chega a $78,7 \%$. O procedimento de partição da variabilidade mostrou que as épocas de coleta explicaram 10,3\% da variabilidade dos dados da comunidade da macrofauna do solo, enquanto que os diferentes manejos de colheita explicaram $6,1 \%$. O teste de Monte-Carlo revelou relações significativas entre os grupos da fauna e as variáveis ambientais testadas tanto para o primeiro eixo canônico, como para a soma de todos os eixos canônicos $(p<0,05)$. Ou seja, embora existam outros fatores que determinam a comunidade da macrofauna, as variáveis testadas tem efeitos significativos sobre os padrões da comunidade. Utilizando-se a regra do biplot para interpretação do diagrama de ordenação, observa-se a relação positiva da abundância de Oligochaeta com 
a amostragem realizada em julho que, contrariamente ao esperado, teve maior precipitação. A amostragem de fevereiro aparece isolada e em sentido inverso ao vetor de julho, demonstrando padrões diferentes entre as épocas. Em relação ao manejo, as setas de CQ e CC-Q são quase coincidentes, levando à interpretação de padrões de comunidades similares.
CQ-C e CC apresentam-se associados a um maior número de grupos. Em particular $\mathrm{CC}$ e a variável riqueza, apresentam um pequeno ângulo entre os vetores, indicando que este ambiente apresenta elevada riqueza média.

Figura 4. Diagrama de ordenação da análise de redundância entre os grupos da macrofauna do solo (setas pontilhadas) e as variáveis ambientais (setas contínuas) em área de cultivo de cana-de-açúcar. Os grupos da macrofauna do solo são: Ara (Araneae), Bla (Blattodea), C Oli (casulos de Oligochaeta), Chi (Chilopoda), Col (Coleoptera adultos), For (Formicidae), Gas (Gastropoda), Het (Heteroptera), Iso (Isoptera), LCol (larvas de Coleoptera), Oli (Oligochaeta), Riq (número médio de grupos). As variáveis ambientais são: épocas de amostragem (Fevereiro e Julho) e manejo da colheita da cana-de-açúcar, sendo CC (cana crua), CQ (cana queimada), CC-Q (alternância de cana crua com queimada) e CQ-C (alternância de cana queimada com crua).

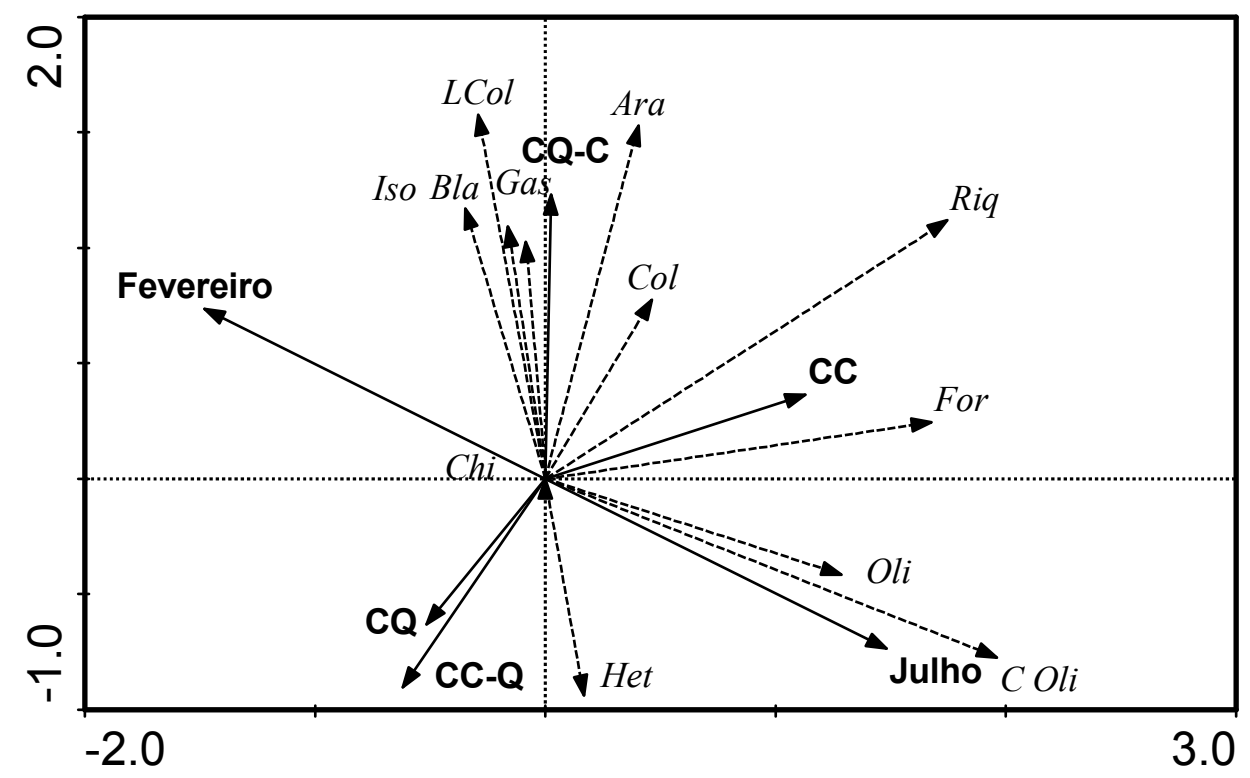

Fonte: Elaboração dos autores.

\section{Conclusões}

A colheita da cana-de-açúcar sem a queima beneficia, diretamente, a riqueza de grupos da macrofauna, oferecendo condições favoráveis à atividade desses organismos e ao surgimento de outros grupos adaptados ao ambiente.

O índice ecológico de riqueza foi mais apropriado e eficiente na avaliação da influência dos sistemas de colheita do que a densidade média total.
Para as duas épocas de amostragem, os baixos valores verificados para os índices de equitabilidade e diversidade são devidos às altas densidades do grupo Formicidae.

A ocorrência de predadores nos tratamentos sem queima da cana-de-açúcar configura um ambiente estável ao estabelecimento de uma teia trófica. 


\section{Agradecimentos}

Usina LASA, técnicos do Campus Dr. Leonel Miranda (UFRRJ), equipe do laboratório de Fauna do Solo - Embrapa Agrobiologia.

\section{Referências}

ALMEIDA FILHO, A. J. Impacto ambiental da queima controlada da cana-de-açúcar sobre a entomofauna. 1995. Tese (Doutorado em Ciências Biológicas) - Escola Superior de Agricultura Luiz de Queiroz. Universidade de São Paulo, Piracicaba.

ALVES, M. V.; SANTOS, J. C. P.; GOIS, D. T.; ALBERTON, J. V.; BARETTA, D. Macrofauna do solo influenciada pelo uso de fertilizantes químicos e dejetos de suínos no oeste do estado de Santa Catarina. Revista Brasileira de Ciência do Solo, Viçosa, v. 32, n. 2, p. 589598, 2008.

ANDERSEN, A. N.; HOFFMANN, B. D.; MÜLLER, W. J.; GRIFFITHS, A. D. Using ants as bioindicators in land management: simplifying assessment of ant community responses. Journal of Applied Ecology, London, v. 39, n. 1, p. 8-17, 2002.

ANDERSON, J. M.; INGRAM, J. S. I. Tropical soil biological and fertility: a handbook of methods. 2. ed. Wallingford: CAB International, 1993. $221 \mathrm{p}$.

AQUINO, A. M. de; SILVA, R. F. da; MERCANTE, F. M.; CORREIA, M. E. F.; GUIMARÃES, M. F.; LAVELLE, P. Invertebrate soil macrofauna under different ground cover plants in the no-till system in the Cerrado. European Journal of Soil Biology, Braunschweig, v. 44, n. 2, p. 91-91, 2008.

ARAÚJO FILHO, J. A.; BARBOSA, T. M. L. Sistemas agrícolas sustentáveis para regiões semi-áridas. Sobral, CE: EMBRAPA Caprinos, 2000. 18 p. (Circular técnica, 20).

ASSAD, M. L. L. A fauna do solo. In: VARGAS, M. A. T.; HUNGRIA, M. (Ed.). Biologia dos solos dos cerrados. Planaltina: Embrapa/CPAC, 1997a. p. 363-443.

Papel da macrofauna edáfica de invertebrados no comportamento de solos tropicais. In: CONGRESSO BRASILEIRO DE CIÊNCIAS DO SOLO, 26., Rio de Janeiro. Conferências... Rio de Janeiro: SBCS, 1997b. CD-ROM.

BARETTA, D.; MAFRA, A. L.; SANTOS, J. C. P.; AMARANTE, C. V. T.; BERTOL, I. Análise multivariada da fauna edáfica em diferentes sistemas de preparo e cultivo do solo. Pesquisa Agropecuária Brasileira, Brasília, v. 41, n. 11, p. 1675-1679, 2006.
BARRIOS, E.; COBO, J. G.; RAO, I. M.; THOMAS, R. J.; AMEZQUITA, E.; JIMENEZ, J. J.; RONDON, M. A. Fallow management for soil fertility recovery in tropical Andean agroecosystems in Colombia. Agriculture, Ecosystems and Environment, Amsterdam, v. 110, n. 1-2, p. 29-42, 2005.

BARROS, E.; GRIMALDI, M.; SARRAZIN, M.; CHAUVEL, A.; MITJA, D.; DESJARDINS, T.; LAVELLE, P. Soil physical degradation and changes in macrofaunal communities in Central Amazon. Applied Soil Ecology, Amsterdam, v. 26, n. 2, p. 157-168, 2004.

BATÁRY, P.; BÁLDI, A.; SAMU, F.; SZÜTS, T.; ERDÖS, $\mathrm{S}$. Are spiders reacting to local or landscape scale effects in Hungarian pastures? Biological Conservation, Essex, v. 141, n. 8, p. 2062-2070, 2008.

BIANCHI, M. O.; AQUINO, A. M.; ALMEIDA, E. Distribuição vertical da macrofauna do solo em várias safras do milho agroecológico em área do produtor familiar. In: CONGRESSO BRASILEIRO DE CIÊNCIA DO SOLO, 31., 2007, Gramado. Anais... Gramado: Sociedade Brasileira de Ciência do Solo, 2007. CDROM.

BLANCHART, E.; VILLANAVE, C.; VIALLATOUX, A.; BARTHÈS, B.; GIRARDIN, C.; AZONTONDE, A.; FELLER, C. Long-term effect of a legume cover crop (Mucuna pruriens var. Utilis) on the communities of soil macrofauna and nematofauna, under maize cultivation, in southern Benin. European Journal of Soil Biology, Braunschweig, v. 42, p. 136-144, 2006. Supplement 1

BRASIL. Ministério da Agricultura, Pecuária e Abastecimento. Culturas: cana-de-açúcar. Secretaria de Produção e Agroenergia (SPAE). Brasília, 2012. Disponível em: <http://www.agricultura.gov.br/vegetal/ culturas/cana-de-acucar>. Acesso em: 03 nov. 2012.

BRUYN, L. A. L. de. Ants as bioindicators of soil function in rural environments. Agriculture, Ecosystems and Environment, Amsterdam, v. 74, n. 1-3, p. 425-441, 1999.

CAMPANHOLA, C. Compromissos internacionais: convenção sobre diversidade biológica. In: MANZATTO, C. V.; FREITAS JUNIOR, E.; PERES, J. R. R. (Ed.). Uso agrícola dos solos brasileiros. Rio de Janeiro: EMBRAPA Solos, 2002. p. 135-144.

CEDDIA, M. B. Implicações técnicas e alterações fisicicas provocadas pelo sistema de corte da cana-de-açúcar em solo de tabuleiro no Espírito Santo. 1996. Dissertação (Mestrado em Agronomia) - Universidade Federal Rural do Rio de Janeiro, Seropédica. 
COLLETT, N. G. Effects of two short rotations prescribed fires in autumn on surface-active arthropods in dry sclerophyll eucalypt forest of west-central Victoria. Forestry Ecology and Management, Ringwood, v. 107, n. 1-3, p. 253-273, 1998.

CORDEIRO, F. C.; DIAS, F. C.; MERLIM, A. O.; CORREIA, M. E. F., AQUINO, A. M.; BROWN, G. Diversidade da macrofauna invertebrada do solo como indicadora da qualidade do solo em sistema de manejo orgânico de produção. Revista Universidade Rural, Série Ciência da Vida, Seropédica, v. 24, n. 2, p. 29-34, 2004.

CORREIA, M. E. F.; OLIVEIRA, L. C. M. Fauna do solo: aspectos gerais e metodológicos. Rio de Janeiro: Embrapa Agrobiologia, Seropédica, 2000. 46 p. (Documento, 112)

CORREIA, M. E. F.; PINHEIRO, L. B. A. Monitoramento da fauna de solo sob diferentes coberturas vegetais em um sistema integrado de produção agroecológica, Seropédica, (RJ). Seropédica: Embrapa Agrobiologia, 1999. 15 p. (Embrapa-CNPAB. Circular técnica, 3).

DANGERFIELD, J. M.; TELFORD, S. R. Species diversity of julid millipedes: between habitat comparisons within seasonal tropics. Pedobiologia, Jena, v. 36, n. 6, p. 321-329, 1992.

DEVIDE, A. C. P.; CASTRO, C. M. Manejo do solo e a dinâmica da fauna edáfica. Revista de Pesquisa \& Tecnologia, Polo Regional do Vale do Paraíba, v. 5, n. 2, 2008.

DIAS, P. F.; SOUTO, S. M.; CORREIA, M. E. F.; ROCHA, G. P.; MOREIRA, J. F.; RODRIGUES, K. M.; FRANCO, A. A. Árvores fixadoras de nitrogênio e macrofauna do solo em pastagens de híbrido de Digitaria. Pesquisa Agropecuária Brasileira, Brasília, v. 41, n. 6, p. 1015-1021, 2006.

DIDDEN, W. A. M. Ecology of terrestrial Enchytraeidae. Pedobiologia, Jena, v. 37, n. 1-2, p. 2-29, 1993.

DLAMINI, T. C.; HAYNES, R. J. Influence of agricultural land use on the size and composition of earthworm communities in northern KwaZulu-Natal, South Africa. Applied Soil Ecology, Amsterdam, v. 27, n. 1, p. 77-88, 2004.

EMPRESA BRASILEIRA DE PESQUISA AGROPECUÁRIA - EMBRAPA. Centro Nacional de Pesquisa em Solos. Sistema brasileiro de classificação de solos. 2. ed. Rio de Janeiro: EMBRAPA Solos, 2006. $306 \mathrm{p}$.
GIRACCA, E. M. N.; ELTZ, F. L. F.; ANTONIOLLI, Z. I.; WENDLING, A.; VENTURINI, E. F. Macrofauna em solo sob plantio direto com diferentes doses de calcário. In: REUNIÃO BRASILEIRA DE MANEJO E CONSERVAÇÃO DO SOLO E DA ÁGUA, 14., Cuiabá, 2002. Anais... Cuiabá: [s.n.], 2002. Disponível em: $<$ http://w3.ufsm.br/ppgcs/eventos/XIVManejo.php $>$. Acesso em: 16 dez. 2009.

GOSSELIN, F. An assessment of the dependence of evenness indices on species richness. Journal of Theoretical Biology, London, v. 242, n. 3, p. 591-597, 2006.

HÅGVAR, S. Mites (Acari) developing inside decomposing spruce needles: biology and effect on decomposition rate. Pedobiologia, Jena, v. 42, n. 4, p. 358-377. 1998.

INSTITUTO CAPIXABA DE PESQUISA, ASSISTÊNCIA TÉCNICA E EXTENSÃO RURAL - INCAPER. Clima: precipitação. Vitória, 2012. Disponível em: <http://hidrometeorologia.incaper. es.gov.br/?pagina=precipitacao $>$. Acesso em: 08 nov. 2012 .

KITAMURA, A. E.; ALVES, M. C.; SUZUKI, L. G. A. S.; GONZALEZ, A. P. Recuperação de um solo degradado com a aplicação de adubos verdes e lodo de esgoto. Revista Brasileira de Ciência do Solo, Viçosa, v. 32, n. 1, p. 405-416, 2008.

LAVELLE, P. Assessing the abundance and role of invertebrate communities in tropical soils: aims and methods. Journal of African Zoology, Kwazulu-Natal, v. 102, p. 275-283, 1988.

Functional domains in soils. Ecological Research, Tokyo, v. 17, n. 4, p. 441-450, 2002.

LAVELLE, P.; DANGERFIELD, M.; FRAGOSO, C.; ESCHENBRENNER, V.; LOPEZ-HERNANDEZ, D.; PASHANASI, B.; BRUSAARD, L. The relationship between soil macrofauna and tropical soil fertility. In: WILEY, J. \& Sons (Ed.). The biological management of tropical soil fertility. United Kingdom: TSBF and Sayce, 1994. cap. 6, p. 137-170.

LAVELLE, P.; LATTAUD, C.; TRIGO, D.; BAROIS, I. Mutualism and biodiversity in soils. Plant and Soil, Hague, v. 170, n. 1, p. 23-33, 1995.

LEGENDRE, P.; ANDERSON, M. J. Distance-based redundancy analysis: testing multispecies responses in multifactorial ecological experiments. Ecological Monographs, Ithaca, v. 69, n. 1, p. 1-24, 1999.

MAJER, J. D. Ant recolonization of rehabilitated bauxite mines at Trombetas, Pará, Brazil. Journal of Tropical Ecology, Cambridge, v. 12, n. 2, p. 257-273, 1996. 
Short-term responses of soil and litter invertebrates to a cool autumn burn in Jarrah (Eucalyptus marginata) forest in Western Australia. Pedobiologia, Jena, v. 26, n. 4, p. 229-247, 1984.

MARTINHO, A. da F.; LIMA, E.; PEREIRA, M. G.; FERNANDES, M. M.; MALHEIROS. M. G.; ZONTA, E. Mesofauna em Argissolo Amarelo cultivado com cana-de-açúcar sob diferentes manejos. In: FERTBIO, 2004, Lages. Anais... Lages: UDESC, Departamento de Solos/SBCS, 2004. CD-ROM.

MENEZES, C. E. G.; CORREIA, M. E. F.; PEREIRA, M. G.; BATISTA, I.; RODRIGUES, K. M.; COUTO, W. H.; ANJOS, L. H. C.; OLIVEIRA, I. P. Macrofauna edáfica em estádios sucessionais de floresta estacional semidecidual e pastagem mista em Pinheiral (RJ). Revista Brasileira de Ciência do Solo, Viçosa, v. 33, n. 6, p. 1647-1656, 2009.

MERLIM, A. O. Macrofauna edáfica em ecossistemas preservados e degradados de araucária no Parque Estadual de Campos de Jordão, SP. 2005. Dissertação (Mestrado em Ecologia de Agroecossistemas) - Escola Superior de Agricultura Luiz de Queiroz, Piracicaba.

MOÇO, M. K.; GAMA-RODRIGUES, E. F.; GAMARODRIGUES, A. C.; CORREIA, M. E. F. Caracterização da fauna edáfica em diferentes coberturas vegetais na região norte fluminense. Revista Brasileira de Ciência do Solo, Viçosa, v. 29, n. 4, p. 555-564, 2005.

NUNES, L. A. L.; ARAÚJO FILHO, J. A.; MENEZES, R. I. Q. Recolonização da fauna edáfica em áreas de caatinga submetidas a queimadas. Revista Caatinga, Mossoró, v. 21, n. 3, p. 214-220, 2008.

Diversidade da fauna edáfica em solos submetidos a diferentes sistemas de manejo no semiárido nordestino. Scientia Agraria, Curitiba, v. 10, n. 1, p. 43-40, 2009.

PARR, C. L.; ANDERSEN, A. N.; CHASTAGNOL, A.; DUFFAUD, C. Savanna fires increase rates and distances of seed dispersal by ants. Oecologia, Berlim, v. 151, n. 1, p. 33-41, 2007.

PASQUALIN, L. A.; DIONÍSIO, J. A.; ZAWADNEAK, M. A. C.; MARÇAL, C. T. Macrofauna edáfica em lavouras de cana-de-açúcar e mata no noroeste do Paraná - Brasil. Semina: Ciências Agrárias, Londrina, v. 33, n. 1, p. 7-18, 2012.

PINHEIRO, L. B. A. Estudo da macrofauna de solos cultivados com cana-de-açúcar, sob diferentes manejos de colheita - crua e queimada. 1996. Dissertação (Mestrado em Agronomia - Ciência do Solo) - Universidade Federal Rural do Rio de Janeiro, Rio de Janeiro.
RAVELLI NETO, A.; LIMA, E. Caracterização de uma topossequência de solos sobre sedimentos do terciário $e$ quaternário em Linhares-ES. In: CONGRESSO BRASILEIRO DE CIÊNCIA DO SOLO, 21., 1987, Campinas. Programas e resumos... Campinas: SBCS, 1987. p. 166.

ROSSETTO, R.; DIAS, F. L. F.; VITTI, A.; CANTARELLA, H. Manutenção da fertilidade e recuperação dos solos na cultura da cana-de-açúcar. In: CORTEZ, L. A. B. (Org.). Bioetanol de cana-deaçúcar: P\&D para produtividade e sustentabilidade. São Paulo: Edgard Bluncher Ltda., 2010. cap. 3, v. 1, p. 381 403. Disponível em: <http://www.apta.sp.gov.br/cana/ Coletanea.php?cap=3>. Acesso em: 27 ago. 2010.

ROSSI, C. Q.; NOBRE, C. P.; COELHO, C. P.; BENAZZI. E. S.; RODRIGUES, K.; CORREIA, M. E. F. Efeito de diferentes coberturas vegetais sobre a mesofauna edáfica em manejo agroecológico. Revista Brasileira de Agroecologia, Bahia, v. 4, n. 2, p. 13261330, 2009.

ROVEDDER, A. P.; ANTONIOLLI, Z. I.; SPAGNOLLO, E.; VENTURINI, S. Fauna edáfica em solo susceptível à arenização na região Sudoeste do Rio Grande do Sul. Revista de Ciências Agroveterinárias, Lages, v. 3, n. 2, p. 87-96, 2004.

ROZANSKI, A.; SANTOS, J. C. P.; ALVES, M. V.; HAWERRTH, F. J.; TASCA, F. A. Mesofauna edáfica em áreas de campo nativo, mata de araucária e florestas de pinus em diferentes estágios de desenvolvimento. In: FERTBIO, 2004, Lages. Anais... Lages: UDESC, Departamento de Solos/SBCS, 2004. CD-ROM.

SANTOS, S. R. Q.; VITORINO, M. I.; HARADA, A. Y.; SOUZA, A. M. L.; SOUZA, E. B. A riqueza das formigas relacionadas aos períodos sazonais em Caxiuanã durante os anos de 2006 e 2007. Revista Brasileira de Meteorologia, São José dos Campos, v. 27, n. 3, p. 308314, 2012.

SILVA, R. F.; AQUINO, A. M.; MERCANTE, F. M.; GUIMARÃES, M. F. Macrofauna invertebrada do solo sob diferentes sistemas de produção em Latossolo da região do cerrado. Pesquisa Agropecuária Brasileira, Brasília, v. 41, n. 4, p. 697-704, 2006.

SILVA, R. F.; TOMAZI, M.; PEZARICO, C. R.; AQUINO, A. M.; MERCANTE, F. M. Macrofauna invertebrada edáfica em cultivo de mandioca sob sistemas de cobertura do solo. Pesquisa Agropecuária Brasileira, Brasília, v. 42, n. 6, p. 865-871, 2007. 
SILVA, R. R.; BRANDÃO, C. R. F. Formigas (Hymenoptera: Formicidae) como indicadores da qualidade ambiental e da biodiversidade de outros invertebrados terrestres. Biotemas, Florianópolis, v. 12, n. 2, p. 55-73, 1999.

SNYDER, B. A.; HENDRIX, P. F. Current and potential roles of soil macroinvertebrates (earthworms, millipedes and isopods) in ecological restoration. Restoration Ecology, Crawley, v. 16, n. 4, p. 629-636, 2008.

SOCARRÁS, A. V. La vida del suelo: un indicador de su fertilidad. Agricultura Orgânica, La Habana, v. 4, n. 1, p. 12-14, 1998.

SOUZA, Z. M.; PRADO, R. M.; PAIXÃO, A. C. S.; CESARIN, L. G. Sistemas de colheita e manejo da palhada de cana-de-açúcar. Pesquisa Agropecuária Brasileira, Brasília, v. 40, n. 3, p. 271-278, 2005.

STORK, N. E.; EGGLETON, P. Invertebrates as determinants and indicators of soil quality. American Journal of Alternative Agriculture, Greenbelt, v. 7, n. 1-2, p. 38-47, 1992. ter BRAAK, C. J. F.; SMILAUER, P. CANOCO reference manual and canodraw for windows user's guide: software for canonical community ordination (Version 4.5). Microcomputer Power, Ithaca, NY, USA. 2002. $500 \mathrm{p}$.

TOPP, W.; SIMON, M.; KAUTZ, G.; DWORSCHAK, U.; NICOLINI, F.; PRÜCKNER, S. Soil fauna of a reclaimed lignite open-cast mine of the Rhineland: improvement of soil quality by surface pattern. Ecological Engineering, Netherlands, v. 17, n. 2-3, p. 307-322, 2001.

VERHOEF, H.; WITTEVEEN, J. Water balance in Collembola and its relation to habitat selection; cuticular water loss and water uptake. Journal of Insect Physiology, Kidlington, v. 26, n. 3, p. 201-208. 1980.

WANNER, M.; DUNGER, W. Primary immigration and succession of soil organisms on reclaimed opencast coal mining areas in eastern Germany. European Journal of Soil Biology, Montrouge, v. 38, n. 2, p. 137-143, 2002. 
